\title{
Editorial
}

\section{Publication will be quicker}

There is good news for our contributors concerning the time taken for their accepted papers to be published. Many of them have felt, quite justifiably, that the time lag has been too long for the last 18 months. We should like to explain how this came about, and why the situation is rapidly improving.

The number of papers submitted to the Archives has steadily risen. In 1970 it was roughly 300 per year; by 1979 it was 650 with the average quality higher. The policy of the journal had always been to accept those papers which the editors believed worth publishing without particular attention to numbers. Until recently it has been possible to do this without accumulating an excess of material awaiting publication. We were helped by an increase in the number of printed pages in the early 1970 s, and by the change to monthly publication in 1974. However, in 1978 and 1979 the flow of good papers began to exceed the number we could publish, and our traditional policy of trying to accept them all led to a steady increase in the time interval between acceptance and publication.

This was a matter of great concern to the editors, as well as to our contributors and readers. During the past year we have therefore been exceptionally stringent, and accepted fewer papers than we have published in order to reduce the publication delay. It has been a painful period for us (we dislike turning down papers we want to publish) and for authors who have had sound papers unexpectedly refused-or waited far too long to see their accepted manuscripts appear it print.

However this harsh policy, combined with a suspension of the printing of abstracts (other than those of the British Paediatric Association) and a more selective attitude to correspondence, is now producing results. The publication delay for accepted papers is becoming shorter each month; by the end of the year it will be close to our normal figure of 6 months. We shall also be able to accept a rather higher proportion of the worthy papers, although the rising interest in paediatrics (shown both by the number and quality of papers we receive) will inevitably make it more difficult to get papers in the journal than it was, say, 5 years ago. We thank our authors for the forbearance that the great majority of them have shown during this difficult period and we are glad to be able to tell them that happier times are arriving.

The publication delay for Short Reports is less than for longer original papers, and if there are strong reasons why results should be reported very quickly we can publish a Short Report accepted today in under 6 months. Authors who can abbreviate their papers to not more than 1000 words, 2 tables or figures, and 8 references will therefore benefit by having theirs in print sooner-and we have no doubt that Short Reports are at least as popular with readers as longer articles. 